\title{
Generation of atmospheric wavefronts using binary micro-mirror arrays
}

\author{
Esdras Anzuola and Aniceto Belmonte \\ Technical University of Catalonia, BarcelonaTech, Department of Signal Theory and Communications, 08034 Barcelona, Spain
}

Compiled January 26, 2016

\begin{abstract}
To simulate in the laboratory the influence that the turbulent atmosphere has on light beams, we introduce a practical method for generating atmospheric wavefront distortions that considers digital holographic reconstruction using a programmable binary micro-mirror array. We analyze the efficiency of the approach for different configurations of the micro-mirror array and demonstrate the benchtop technique experimentally. Though the mirrors on the digital array can only be positioned in one of two states, we show that the holographic technique can be used to device a wide variety of atmospheric wavefront aberrations in a controllable and predictable way for a fraction of the cost of a phase-only SLM. ๑ 2016 Optical Society of America
\end{abstract}

OCIS codes: (110.0115) Imaging through turbulent media, (010.1330) Atmospheric turbulence, (090.1760) Computer holography, (90.1000) Aberration compensation.

http://dx.doi.org/10.1364/ao.XX.XXXXXX

\section{INTRODUCTION}

In optical imaging, sensing, and communication systems in the atmosphere, the phases and amplitudes of the received optical fields are affected by atmospheric turbulence in ways that reduces the quality of the detected optical power. Coherent receiving techniques, where a perfect local oscillator beam superimposes the received beam, are particularly vulnerable to turbulence as the unmatched phases and amplitudes of the two beams cause a reduced down-converted power. As a consequence, to maximize the down-converted coherent power and mitigate atmospheric turbulence effects in the performance of the coherent receivers, free space optical systems (FSO) use adaptive optics (AO) such as deformable mirrors for phase compensation [1, 2].

The development of $\mathrm{AO}$ systems is a complex discipline encompassing many challenging problems and, in general, a source that introduces optical aberrations which presents characteristics close to the atmospheric turbulence becomes suitable in the laboratory. These wave-front sources allow us to carry out a performance analysis of AO systems without the necessity of a real atmospheric link, saving telescope time and not being limited by the specific atmospheric condition at a certain time. Also, these systems may be integrated in a functional AO setup and they can be used to perform the calibrations and maintenance required in mounted telescopes. Different methods to generate atmospheric aberrations have been proposed, including wind chambers [3], phase screens [4], moving plates [5] and spatial light modulators (SLMs) [6, 7]. In practice, these methods fulfill just some of the characteristics that an ideal turbulence generator should present in terms of creating dynamic and deterministic wave-front aberrations working at a sufficient spatial resolution and temporal rate to emulate atmospheric disturbances in the receiving light.

In this paper we present a practical turbulence generation technique based on well-known digital micro-mirrors devices (DMDs). The main characteristic of these DMDs is that they are able to modulate the amplitude of the incoming beam only in two levels, zero or one, depending on the state ("on" or "off") of each micro-mirror. The binary nature of the device increases the simplicity of the electronics involved, which results into an increased speed of the reconfiguration rates and reduces the cost to a fraction of the price of typical SLMs [8]. It also presents a wide optical spectral bandwidth, good spatial resolution, and high power damage thresholds, which make it very useful for many light-control applications $[9,10]$.

DMDs are by definition amplitude-modulation devices with reduced capabilities due to the binary nature of the micro-mirror array. To overcome this limitation, DMDs can be paired with binary computer-generated holographic techniques to provide wavefront phase modulation through binary amplitude modulation [11]. In these techniques, the light beam coming from an optical source is separated into different spatial modes by interfering it with a holographic pattern composed by a set of binary gratings. By selecting the first diffracted mode, the phase information recorded onto the holographic pattern is retrieved. The combination of binary computer-generated holographic methods with DMD technology has been recently proposed and successfully validated in several optical setups $[8,12]$. 
In this paper we study the feasibility of generating atmospheric aberrations by using binary digital holography and commercially available DMDs. In Sections 2 and 3, we develop the theoretical background behind the application of binary computer-generated holographic techniques with micro-mirror arrays, identifying the required assumptions and input parameters. Then, in Section 4, we consider the experimental implementation of the technique and detail the different trade-off of the method. In doing so, we try to highlight the main advantages and weaknesses of the wavefront generation technique. We demonstrate experimentally its practicability by creating distorted wavefronts that emulate atmospheric propagation effects.

\section{METHOD OVERVIEW}

Holography is a technique that can record the three dimensional image of an object on an interferogram by using a reference laser beam and the reflected light coming from this object. The image is reconstructed by illuminating the recorded interference fringes with a coherent source and isolating the first diffracted order [13]. In digital holography, the acquisition of holograms can be done with a digital sensor array, typically a CCD camera, and image data reconstruction is performed numerically from digitized interferograms. With modern computer-generated digital holography, there is no acquisition of the holographic image, as it is be generated by digitally computing a holographic interference pattern, and the holographic image data can be rendered by a holographic display operating on the basis of interference of coherent light.

Our digital, computer-generated holograms differ from a normal hologram in that the interferogram fringes needs to have binary amplitude -as the synthesized interferogram fringes are fed to the DMD micro-mirrors, the amplitude of one pixel can only be set to 0 or $1-$ with no control on the phase. Here, we present how digital, computer-generated holography and, in particular, those types that have only a binary interferogram, offers a means of delivering optical phase data images [11]. With these constraints, to get phase modulation using binary amplitude modulation, we need to filter an amplitude signal in the Fourier plane to obtain a complex optical field modulation.

A well-known technique to generate binary holograms is to create a interferogram $h(x, y)$ composed by rectangular fringes in which the position and width of each fringe encode the phase and amplitude, respectively, of the desired field [11]. We can create any desired field $A(x, y) \exp (j \phi(x, y)$ acting over the position and width of the fringes in the interferogram $h(x, y)$ according to $[14,15]$

$$
h(x, y)=\operatorname{sign}\{\cos (2 \pi \alpha+2 \pi \delta(x, y)-\cos (\pi q(x, y))\}
$$

where the parametric functions $\delta(x, y)$ and $q(x, y)$

$$
q(x, y)=\frac{\arcsin \{A(x, y)\}}{\pi}, \quad \delta(x, y)=\frac{\phi(x, y)}{2 \pi},
$$

correspond to the desired amplitude and phase modulation, respectively. Conveniently, Eq. 1 defines a binary amplitude hologram by thresholding the amplitude interferogram with the sign function.

We see from Eq. 2 that $\delta(x, y)$ describes the relative location of each fringe in the interferogram and $q(x, y)$ is a parametric function that controls the corresponding fringe width. The resulting grating $h(x, y)$ consist of a set of rectangular fringes with period $T=\lambda / \alpha$, width $q T$, and a relative location $\delta$ within each period. In Eq. 1, $\alpha$ is a linear tilt, which acts as a spatial carrier

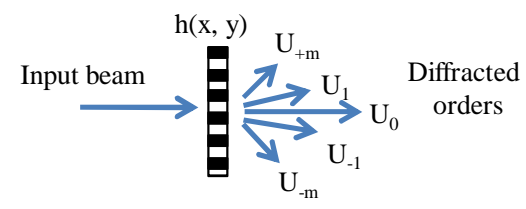

Fig. 1. Binary holography representation: a binary periodic grating diffracts the incoming light-wave into multiple orders.

frequency along the $x$ direction. In effect, by expanding Eq. 1 into a spatial Fourier series

$$
\begin{aligned}
h(x, y) & =\sum_{m} U_{m} \\
& =\sum_{m} \frac{\sin \{\pi m q(x, y)\}}{\pi m} \exp (j 2 \pi m(\alpha+\delta(x, y)))
\end{aligned}
$$

we observe that the terms $U_{ \pm 1}$ (see Fig. 1) corresponds to the desired phase and amplitude modulation tilted by an angle corresponding to the diffraction. By selecting only one of these terms -one can use a 4-f system with an iris in the Fourier plane-a clear representation of the optical field can be obtained.

In Eq. 2, as we focus on phase wavefront aberrations $\phi(x, y)$, and amplitude wavefront distortions $A(x, y)$ are not considered, we are free to fix the parametric function $q(x, y)$ to a constant value $q(x, y)=q_{0}$ and do it in a way that maximizes the diffraction efficiency of the most relevant modes $U_{ \pm 1}$. By choosing $q_{0}=1 / 2$, all the modes of order even in Eq. 3 become zero, and the diffraction efficiency of the modes $U_{ \pm 1}$ reach a maximum of around $10 \%$ [16].

In [17], the statistics of the phase wavefront $\phi$ affected by aberrations induced by atmospheric turbulence were considered using a Kolmogorov description of the random media [18] where Gaussian phase fluctuations are distinctively characterized by its statistical variance $1.0299\left(D / r_{0}\right)^{5 / 3}$. Here, we only need to consider the aperture diameter $D$ of the receiving system normalized by the wavefront coherent diameter $r_{0}[19]$, which describes the spatial correlation of phase fluctuations in the lens pupil plane. Although it would be possible to consider the effects of the inner and outer scales of turbulence in the synthetized wavefronts, in this study we have limited our analysis to simple Kolmogorov phase statistics.

In that classical analysis [17], it was shown that Zernike polynomials $Z_{m}$ of order $m$ can be used as basis functions to describe atmospheric phase wavefront distortions, which is very convenient because of their correspondence to classical aberrations and their simple analytical expressions. Let $\phi(x, y)$ be the atmospheric random phase wavefront to be synthetized with our holographic technique:

$$
\phi(x, y)=\sum_{m=0}^{M} a_{m} Z_{m}(x, y)
$$

Here, the parameter $a_{m}$ is a Gaussian random variable whose variance only depends on the turbulence ratio $\left(D / r_{0}\right)$. For a large number of modes $M$, the error in this phase wavefront representation becomes negligible [17]. In this work, using Eqs. 1-3, we synthetize binary interference patterns for the different Zernike polynomials $Z_{m}$ and combine them according to Eq. 4 to describe atmospherically distorted phase wavefronts.

Interestingly, Eq. 4 can be extended to consider modal compensation of atmospheric phase distortion. Adaptive optics compensation of atmospheric wave-front phase distortion to 
improve the performance of atmospheric systems has been an important field of study for many years. In such optical compensation, it is known that we only need to consider the residual phase variance after modal compensation of a finite number $J$ of Zernike terms. In this adaptive systems, it is desirable to choose the compensation order $J$ large enough that the residual phase $\phi=\sum_{m=J}^{M} a_{m} Z_{m}$ reduces to a minimum.

\section{PERFORMANCE ANALYSIS OF THE METHOD}

In this Section, we put together the physical limitations of the device micromirrors and the requirements imposed by the correct emulation of atmospheric effects on the phase wavefront. We need to evaluate the impact of various parameters, including the ratio $\left(D / r_{0}\right)$ and the number of modes $M$ used in the wavefront representation, in the performance of the DMD-based holographic technique.

The discrete nature of the DMD implies that the interferogram $h(x, y)$ needs to be discretized in the $x$ and $y$ axes in order to be recorded onto a $N_{x} \times N_{y}$ pixel resolution DMD. To generate an exact periodic grating on a DMD, the grating period must be an integer number of pixels $N$. Each grating period can only contain one phase value or symbol, so the original phase wavefront must be re-sampled with period $T=N d x_{p}$, where $d x_{p}$ is the DMD pixel size. At the same time, the relative location of each fringe can only take one of $N$ different values, which limits the generated phase to $N$ different levels. The generated phase wavefront $\left.\phi[k, p]\right|_{N}$ is then a re-sampled and quantizated version of the original phase map. Here, $k$ is the symbol index in the $x$ axis, taking values from 1 to $N_{x} / N$, and $p$ is the symbol index in the $y$ axis, taking values from 1 to $N_{y}$. At the same time, the parameter $N$ defines the resulting angular separation between modes as $\alpha=\lambda / T=\lambda / N d x_{p}$.

Selecting an optimal parameter $T$ will fix the characteristics of the resulting wave-front. A high number of pixels per grating period will diminish the separation between the diffracted orders and the spatial bandwidth that can be generated due to the lower number of phase symbols in which $\phi(x, y)$ is re-sampled. On the other hand, a low number of pixels per period will result in higher quantization errors when encoding the phase but, as the re-sampling rate is increased, the spatial bandwidth available is enhanced. We can conclude that it exists a trade-off between generating high resolution aberrations and high spatial frequency aberrations, so the parameter $T$ has to be chosen depending on the characteristics of each application.

The device under test in this work is a $608 \times 684$ resolution DMD from Texas Instruments (TI DLP3000 DMD) with a micromirror size of $7.5 \mu \mathrm{m}$ and a mirror reconfiguration rate up to 4 $\mathrm{KHz}$, much faster than a typical phase only SLM [8]. Although this problem is not considered in this study, at this point we need to note that, in some applications, spatio-temporal phase wavefront shaping -the combination of shaping in the spectral and spatial domains-needs to be contemplated. In practice, any wavefront synthetizer must have an operating frequency that is higher than the bandwidth of the atmospheric phase perturbations as the rate at which phases must be adjusted will be dictated by the rate at which the atmospheric turbulence fluctuates, generally around $1 \mathrm{kHz}$. One of the main advantages of using a DMD compared with a SLM is that the random phase wavefronts at the receiver plane could change at higher temporal rates. Higher frame rates corresponds to faster fluctuations of the atmospheric channel and, to date, the highest frequency regular SLMs can only operate at frequencies of about $100 \mathrm{~Hz}$,

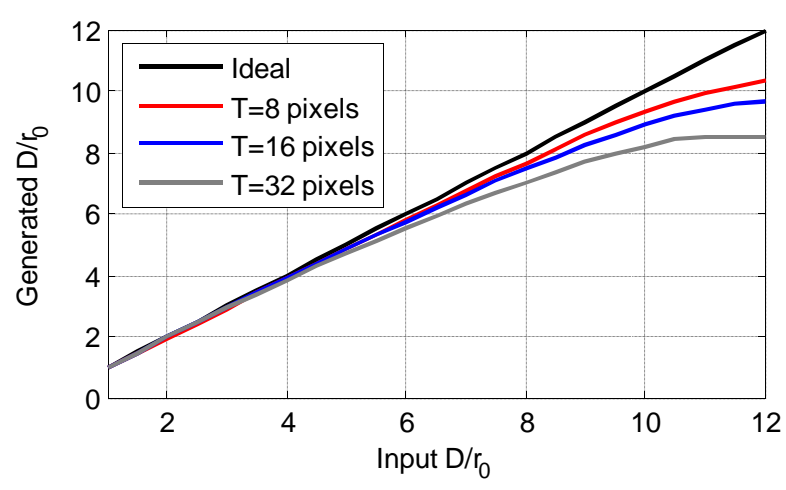

(a)

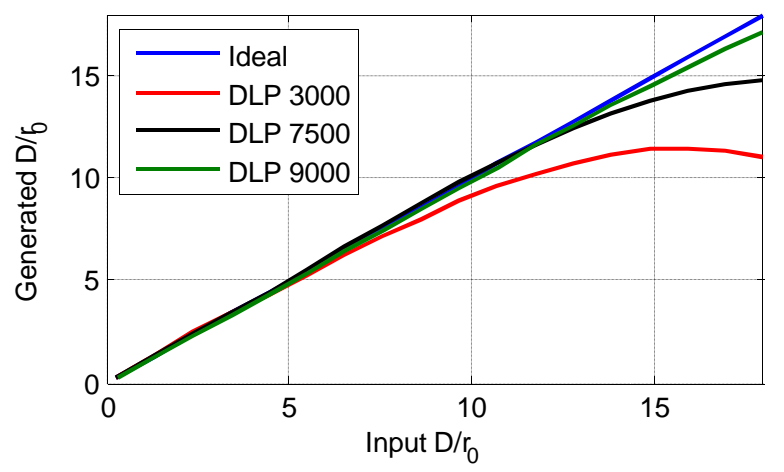

(b)

Fig. 2. Aperture diameter $D$ normalized by the wavefront coherence diameter $r_{0}$ describing the spatial correlation of phase fluctuations in the receiver plane. (a) The input $\left(D / r_{0}\right)$ ratio is compared to the effective $\left(D / r_{0}\right)$ ratio synthetized with a DMD 3000 TI using different grating periods T. (b) For a fixed grating period $T=8$, input $\left(D / r_{0}\right)$ are compared to effectively synthetized $\left(D / r_{0}\right)$ with different commercially available DMDs.

at least one order of magnitude smaller than achievable with DMDs.

In Fig. 2 the normalized aperture diameter $\left(D / r_{0}\right)$ generated by the DMD is shown for each grating period and turbulence strength scenario. It is shown that the effectiveness of the method is reduced when the ratio $\left(D / r_{0}\right)$, i.e., the atmospheric turbulence strength, increases. The reason is that, as the spatial bandwidth of the wave-front increases, the re-sampling process performed by the hologram progressively introduces higher aliasing effects. As it can be seen in Fig. 2(a), a grating period $T$ of 8 pixels presents the best response among the options considered in order to reproduce a wide set of normalized turbulence strength scenarios. In Fig. 2(b) the generated $\left(D / r_{0}\right)$ can be seen for different commercially available DMDs for different resolution parameters and a grating period of 8 pixels. It is expected that better performance could be achieved by using newer generations of DMDs that are available with higher resolution characteristics. We show that just by upgrading the $\mathrm{DMD}$ we can generate a wider set of generation scenarios. Here, along with DLP3000, we consider DLP7000 and DLP9500. By using the DLP3000 we can achieve perturbation scenarios up to $\left(D / r_{0}\right)<10$ with a generation accuracy above $90 \%$. The DLP7000 presents a higher resolution of $1024 \times 768$ pixels, which 
allows us to generate perturbations up to $\left(D / r_{0}\right)<15$. Thanks to its $1920 \times 1080$ pixel resolution, the DLP9500 is able to synthetized efficiently wavefronts with $\left(D / r_{0}\right)$ ratios up to 18 .

\section{EXPERIMENTAL SET-UP AND METHOD VERIFICA- TION}

In order to demonstrate the functionality of the micromirrorbased holographic method, an experimental setup have been implemented around the TI DLP3000 DMD. We have inserted the wavefront generator into a Mach-Zehnder interferometer to image and verify that the phase wave-fronts are being correctly synthetized.

The experimental set-up can be seen in Fig. 3(a). First, a 1550 nm collimated Gaussian beam coming from the light source, which presents a beam width that matches the DMD effective area diameter $(3.41 \mathrm{~mm})$, is divided into a reference beam and a signal beam. The signal beam hits the DMD, which can reflect the incoming light into two different angular positions, i.e., $12^{\circ}$ (on state) and $-12^{\circ}$ (off state) [20]. Then, the resulting first spatial mode $U_{1}$ is filtered using a 4 -f system with a pinhole in the Fourier plane. Considering the maximum achievable power efficiency provided by the binary holographic technique $(10.1 \%$, as explained above), the effective reflectivity of the mirrors $(40 \%$ at the 1550-nm working wavelength), and some DMD residual modulation that diffracts part of the incoming light into spurious modes [20], the selected first diffraction mode concentrates just around $1 \%$ of the incident power. Finally, the reference beam is made to interfere with the selected mode reflected from the DMD using a beam splitter. The interference power pattern is recorded using a CCD camera.

The atmospheric aberrations are generated by loading the computer generated binary holographic patterns onto the DMD. The first twelve Zernike modes were introduced into the DMD and interference patterns were obtained by using the MachZehnder interferometer. Fig. 3(b) shows the resulting phase maps. The phase map profiles match the wave-front aberration and verify that the wave-fronts are generated correctly. Fig. 3(c) shows three examples of the desired atmospherically distorted wavefronts as seen by the Mach-Zehnder interferometer. Three different illustrative $\left(D / r_{0}\right)$ ratios have been considered $(3,5$, and 7). In these examples, we have combined $M=250$ Zernike modes using Eq. 4 and send the corresponding binary pattern onto the DMD. These results are qualitatively very appealing.

To obtain a more quantitative verification of the method, we have implemented in the lab a free-space fiber coupling system that considers adaptive optics compensation. In many atmospheric optical systems, the received laser beam must be coupled into a single-mode optical fiber at the input of the receiver module. However, propagation through atmospheric turbulence degrades the spatial coherence of a laser beam and limits the fiber-coupling efficiency -as usual, coupling efficiency is expressed here as the ratio of the available power at the input of the coupling system, to the power effectively transferred to the single-mode optical fiber [21, 22]. Remarkably, a considerable improvement of the coupling efficiency is expected using adaptive optics in the system that can correct (at least partially) the effects of turbulence.

Here, we have used our source of atmospherically distorted wavefronts to synthetize phase wavefront residual phase $\phi=$ $\sum_{m=J}^{M} a_{m} Z_{m}$ considering different levels of adaptive modal compensation $J$ and carry out a performance analysis of the adaptive coupling system without the necessity of a real atmospheric link.

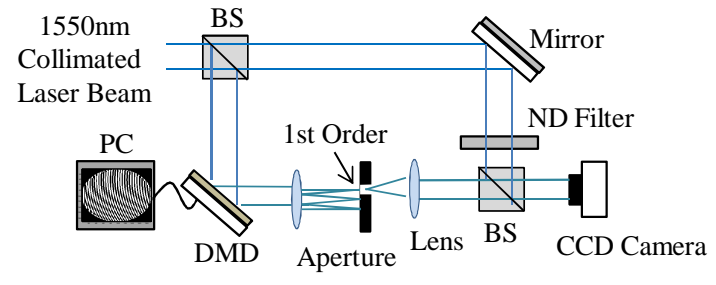

(a)
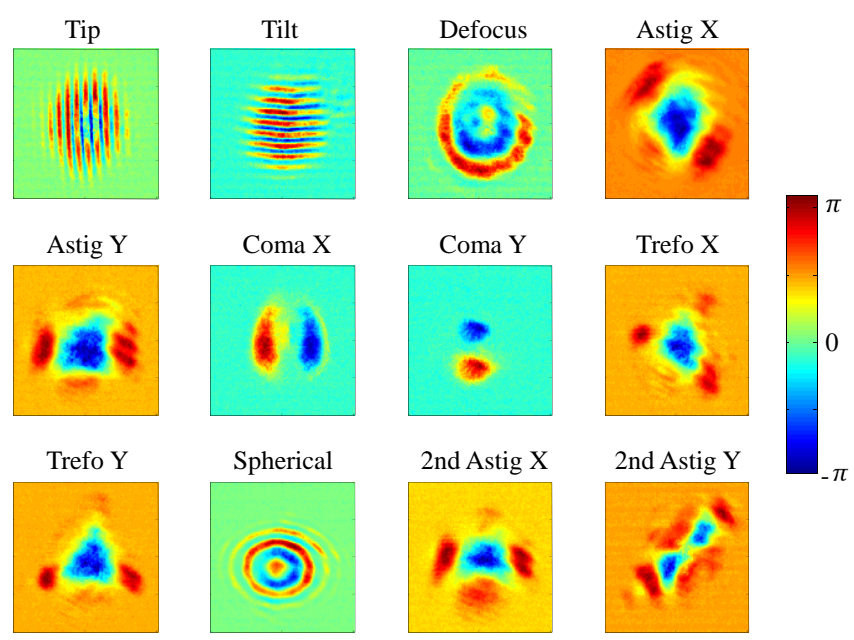

(b)
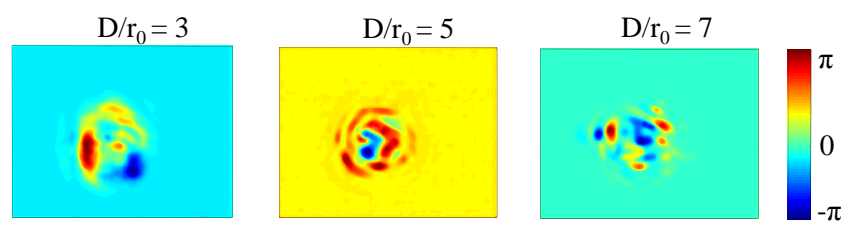

(c)

Fig. 3. (a) Experimental Mach-Zehnder interferometric set-up. (b) Measured interference patterns using the Mach-Zehnder interferometer for the first twelve Zernike modes synthetized by the DLP3000 DMD. (c) Measured interference patterns for synthetized atmospheric phase wavefronts with different atmospheric turbulence conditions $\left(D / r_{0}\right)$.

After evaluating numerically the expected fiber-coupling efficiency for laser light distorted by atmospheric turbulence, and later compensated by adaptive optics, we compare these estimations with experimental measurements for different number of modes $J$ removed by adaptive optics.

In our experiments, the residual phase wavefronts are estimated after compensation of atmospheric aberrations up to tip, astigmatism, coma and fifth-order -corresponding to $J=$ $2,6,10$ and 20, respectively -and the synthetized binary interferograms are sent into the DMD. Different $\left(D / r_{0}\right)$ ratios have been analyzed in the lab. For each $J$ and $\left(D / r_{0}\right)$ values a set of 100 independent phase wavefronts are synthetized and the power coupled into the single-mode fiber is measured for each of them using the experimental set up shown in Fig. 4(a). Here, a 1550-nm collimated Gaussian beam is sent through a free space optical circulator that redirects the light perpendicularly to the DMD, maximizing the effective area of the TI 3000DMD. The first reflected mode is then isolated using a 4 -f system . Finally, 


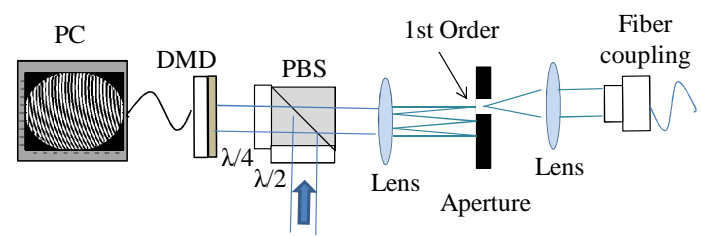

(a)

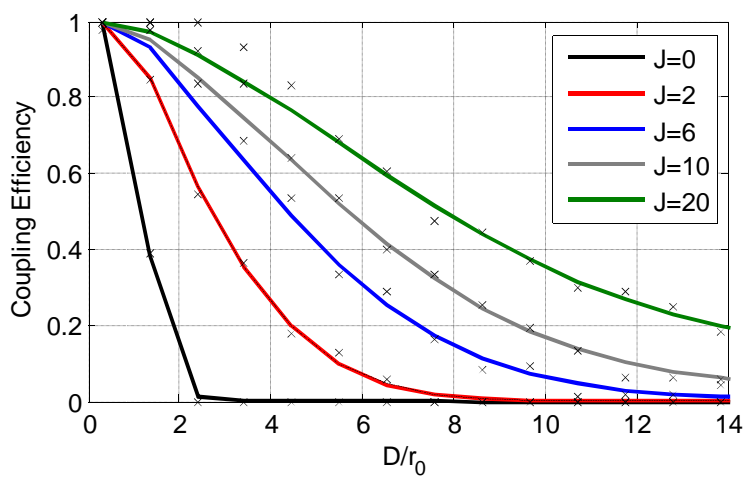

(b)

Fig. 4. Wavefront generator characterization: (a) experimental set-up and (b) theoretical (color line) and measured (cross marks) coupling efficiency of a distorted wavefront into a single mode fiber when $J$ modes are removed by adaptive optics.

the aberrated wavefront is coupled into a single-mode optical fiber using a $2.97 \mathrm{~mm}$ focal aspheric lens. The coupled power is measured at the end of the fiber with a optical power meter.

In Fig. 4(b), the numerical (color line) and measured (cross marks) coupling efficiencies are compared. Small deviations between the theoretical and measured efficiencies are present for $\left(D / r_{0}\right) \geq 10$ due to the DMD limitations. These deviations are consistent with the results obtained in Fig. 2, which imposed an upper level in the coherence length that the DMD is able to reproduce. In any case, for values below that threshold, the fiber-coupling efficiency measured in the lab achieve values remarkably close to the theoretical ones. These results validate the effectiveness of the proposed technique to generate atmospheric wavefronts using binary micro-mirror arrays.

\section{CONCLUSIONS}

In this work, we introduce a practical method for generating atmospheric wavefront distortions that considers digital holographic techniques on a programmable binary micro-mirror array. The technique uses Zernike polynomials and considers Kolmogórov statistics. We study the accuracy of the method for different apertures, devices and perturbation scenarios. We analyze the efficiency of the approach for different configurations of the micro-mirror array and demonstrate the benchtop technique experimentally.

This technology presents the potential of emulating the atmospheric turbulence in a controllable and predictable way at a fraction of the cost of a SLM while providing good performance under a wide range of turbulence strength scenarios and fast reconfiguration rates. The micromirror device used in our experiments is able to emulate atmospheric turbulence up to $\left(D / r_{0}\right)=10$ with very high accuracies, but new upgraded versions of the same device have the potential to achieve similar accuracies for $\left(D / r_{0}\right)$ ratios about to 20 .

This research was funded by the Spanish Department of Science and Innovation MICINN Grant No. TEC 2012-34799. The work of E. Anzuola was supported by a Fellowship Grant for doctoral students by the Spanish Department of Education MEC.

\section{REFERENCES}

1. A. Belmonte and J. Khan, "Performance of synchronous optical receivers using atmospheric compensation techniques," Opt. Express 16, 14151-14162 (2008).

2. A. Belmonte, "Capacity of coherent laser downlinks," J. Lightw. Technol. 32, 2128-2132 (2014).

3. O. Keskin, L. Jolissaint, and C. Bradley, "Hot-air optical turbulence generator for the testing of adaptive optics systems: principles and characterization," Appl. Opt. 45, 4888-4897 (2006).

4. D. J. Butler, S. Hippler, S. Egner, W. Xu, and J. Bähr, "Broadband, static wave-front generation: Na-ag ion-exchange phase screens and telescope emulation," Appl. Opt. 43, 2813-2823 (2004).

5. J. Sasian and E. Acosta, "Generation of spherical aberration with axially translating phase plates via extrinsic aberration," Opt. Express 22, 289-294 (2014).

6. T. Xu, Y. Li, W. Du, C. Ma, S. Cai, M. Lan, J. Wu, S. Yu, and J. Lin, "Simulating atmospheric turbulence using a spatial light modulator based on fourier transform," in "Lasers and Electro-Optics (CLEO), 2014 Conference on," (IEEE, 2014), pp. 1-2.

7. J. D. Phillips, M. E. Goda, and J. Schmidt, "Atmospheric turbulence simulation using liquid crystal spatial light modulators," in "Optics \& Photonics 2005," (International Society for Optics and Photonics, 2005), pp. 589406-589406.

8. M. Mirhosseini, O. S. Magana-Loaiza, C. Chen, B. Rodenburg, M. Malik, and R. W. Boyd, "Rapid generation of light beams carrying orbital angular momentum," Opt. Express 21, 30196-30203 (2013).

9. W.-K. Yu, M.-F. Li, X.-R. Yao, X.-F. Liu, L.-A. Wu, and G.-J. Zhai, "Adaptive compressive ghost imaging based on wavelet trees and sparse representation," Opt. Express 22, 7133-7144 (2014).

10. A. Masson, M. Pedrazzani, S. Benrezzak, P. Tchenio, T. Preat, and D. Nutarelli, "Micromirror structured illumination microscope for highspeed in vivo drosophila brain imaging," Opt. Express 22, 1243-1256 (2014).

11. W.-H. Lee, "Binary computer-generated holograms," Appl. Opt. 18, 3661-3669 (1979).

12. V. Lerner, D. Shwa, Y. Drori, and N. Katz, "Shaping laguerre-gaussian laser modes with binary gratings using a digital micromirror device," Opt. Lett. 37, 4826-4828 (2012).

13. M. Otsubo, H. Takami, and M. Iye, "Holographic atmospheric turbulence simulator for testing adaptive optics systems," Publications of the Astronomical Society of the Pacific pp. 1057-1061 (1997).

14. B. Brown and A. Lohmann, "Computer-generated binary holograms," IBM Journal of research and Development 13, 160-168 (1969).

15. W.-H. Lee, "Binary synthetic holograms," Appl. Opt. 13, 1677-1682 (1974).

16. J. A. DeSanto and E. Wolf, Progress in Optics, vol. 23 (Elsevier, 1986).

17. R. J. Noll, "Zernike polynomials and atmospheric turbulence," J. Opt. Soc. Am. A 66, 207-211 (1976).

18. A. N. Kolmogorov, "The local structure of turbulence in incompressible viscous fluid for very large reynolds numbers," in "Dokl. Akad. Nauk SSSR,", vol. 30 (1941), vol. 30, pp. 299-303.

19. D. L. Fried, "Statistics of a geometric representation of wavefront distortion," J. Opt. Soc. Am. A 55, 1427-1431 (1965).

20. T. Instruments, "Using lasers with $d l p \AA$ dmd technology," Lasers and DLP, TI DN 2509927 (2008).

21. P. J. Winzer and W. R. Leeb, "Fiber coupling efficiency for random light and its applications to lidar," Opt. Lett. 23, 986-988 (1998).

22. Y. Dikmelik and F. M. Davidson, "Fiber-coupling efficiency for free-space optical communication through atmospheric turbulence," Appl. Opt. 44, 4946-4952 (2005). 